\title{
An epidemiological MSEIR model described by the Caputo fractional derivative
}

\author{
Ricardo Almeida ${ }^{1}$, Artur M. C. Brito da Cruz ${ }^{1,2}$, Natália Martins ${ }^{1}$, \\ M. Teresa T. Monteiro ${ }^{3}$ \\ ${ }^{1}$ Center for Research and Development in Mathematics and Applications \\ (CIDMA) \\ Department of Mathematics, University of Aveiro, 3810-193 Aveiro, Portugal \\ ${ }^{2}$ Escola Superior de Tecnologia de Setúbal, Estefanilha, 2910-761 Setúbal, \\ Portugal \\ ${ }^{3}$ Algoritmi R\&D Center, Department of Production and Systems \\ University of Minho, Campus de Gualtar, 4710-057 Braga, Portugal
}

\begin{abstract}
A fractional MSEIR model is presented, involving the Caputo fractional derivative. The equilibrium points and the basic reproduction number are computed. An analysis of the local asymptotic stability at the disease free equilibrium is given. Finally, using Matlab, a numerical simulation of the varicella outbreak among Shenzhen school children, China, is carried out.
\end{abstract}

Mathematics Subject Classification 2010: 26A33, 92D30, 37N25 .

Keywords: Fractional calculus, fractional differential equations, epidemics, MSEIR model, local stability.

\section{Introduction}

Infectious diseases, also known as contagious diseases, are disorders caused by microorganisms such as bacteria or virus. Some infectious diseases can be transmitted through bites from insects or from person to person. Others are acquired by exposure to organisms in the environment or by ingesting contaminated food or water. Millions of people die annually from infectious diseases like measles, tuberculosis, HIV/AIDS, or ebola. Infectious diseases are still not eradicated since there are many countries with poor sanitary conditions or lack of medical care, therefore creating favorable conditions to spread the infectious agents. Moreover, in some cases, the pathogens create new strains resistant to current medications potentially leading to new epidemics.

The main goal of mathematical models in epidemiology is to understand the behavior of a particular infectious disease, such as the prevalence and the duration of the epidemic, and its impact in the population. These models can help the health authorities to choose the best strategies when dealing with the epidemic, like mass vaccination programmes, use of antiviral drugs, pest control, disinfections, and enforce the use of isolation and quarantine.

Mathematical models are used to describe reality, but usually they are simplifications because it is almost impossible to make computations with a large set of input parameters. For example, when studying infectious diseases, usually we disregard some parameters such as weather conditions, individuals' diets, other diseases and types of contact between particular individuals, etc. In this sense, it is important to identify the main variables that significantly influence the model in order to decrease the computational complexity and simultaneously to have a good description of the evolution of the disease. 
In general, an epidemiological mathematical model divides the overall population into disjoint compartments where each compartment represents a specific health status with respect to an infectious agent under consideration. The model is dynamic because the number of individuals of each compartment may fluctuate over time when their health status changes. This feature is of the upmost importance in an infectious disease with a short infectious period, such as measles, varicella, rubella or mumps. Typically, in this kind of diseases, the dynamics of birth and death are often omitted because the time frame is a short period.

One of the simplest compartmental models in epidemiology divides the population in three health states (see [5, 9]): Susceptible (S) to the infection of the infectious agent, Infected (I) by the infectious agent and Recovered/Immune $(\mathrm{R})$ to the disease. These models are known as SIR models. The first SIR model was presented by Kermack and McKendrick in 1927 [30] and played a major influence on the development of mathematical models for disease spread. Later on, these authors contributed further to the development of epidemiological models by the introduction of birth and death rates in the problem (see [31,32]). Since then, numerous works have come up with different approaches. For example, the Reed-Frost and Greenwood discrete-time epidemic models [1] and [9], the SI model [66] (that is, once infected there is no recovery), or more complex SIR models. For example, we refer to [19] and [43], where the susceptible population is divided into subgroups with different infection rates, or there may be multiple levels of infections, some lethal, some sublethal [10]. More complex models can be considered to include, for example, antiviral treatment and vaccination policies $[25,26,41]$.

To make these basic epidemiological models into more realistic ones, other compartments can be included. For example, an Exposed (E) compartment, when there is a significant incubation period during which the individual has been infected but has not yet symptoms, and a Maternallyderived immunity $(\mathrm{M})$, when considering newborns that are immune during the first months of life as a result of maternal antibodies. The model that takes into account all of these compartments is referred as the MSEIR model. This scheme is suitable for diseases that confers permanent immunity, like measles, varicella, rubella or mumps. Therefore, the considered classes in the MSEIR model are:

- $M$ : the individuals with passive immunity, protected by maternal antibodies;

- $S$ : the susceptible class, those individuals who can incur the disease but are not yet exposed to the disease;

- $E$ : the individuals exposed to the disease but not yet infectious;

- $I$ : the individuals infected by the disease and transmitting the disease to others;

- $R$ : the recovered, with permanent immunity.

Depending on the classes considered, we have different epidemiological models. For example, the SIS model [27, 69], the SIRS model [34, 42], the SEIR model [35, 39], the MSEIR model [28], among others. Epidemiological models have been successfully applied to the study of several diseases such as HIV/AIDS [52, 68], Ebola [37, 38], Influenza [63], Cancer [2], Dengue [55, 64], Malaria [54, 54], Salmonella infection [57], and Zika [49].

Recently, fractional derivatives have been used to describe epidemiological models and they have proven to be more accurate in some cases, when compared to the classical ones. We find in the literature different models described by fractional derivatives, like the SIR model $[6,7,21$, $24,47,50,60,61]$, the SIR model with vaccination [17, 36, 58], the SIRC model [17], the SEIR model $[23,51]$, the MSEIR model [8], etc. We also refer some applications to biomedical modelling $[11,29,44,45,48]$.

In this paper, we propose a fractional MSEIR model, where the spread of the disease is described by a system of nonlinear fractional-order differential equations. It is worthwhile mentioning that fractional derivatives are non-local operators, and thus may be more suitable for modeling systems dependent on past history (memory). Also, since the fractional order can be any positive 
real $\alpha$, we can choose the one that better fits the data. Therefore, we can adjust the model to real data and, thus, better predict the evolution of the disease.

There are several definitions for fractional derivatives; in this paper we choose to work with the Caputo fractional derivative. One of the advantages of such derivative is allowing us to consider classical initial conditions to be included in the formulation of the problem. Also, the Caputo fractional derivative of a constant is zero, which is not true for other fractional derivatives.

The paper is outlined as follows. In Section 2, we first recall some definitions and results about fractional calculus and also present a new result that will be needed in what follows. In Section 3, we present the classical MSEIR model, and then, in Section 4, extend it to the context of fractional calculus. Namely, we prove that the problem is well-posed and we evaluate the equilibrium points and the basic reproduction number. Numerical simulations using Matlab are given in Section 5, where we study a varicella outbreak in the region of Shenzhen, China, in 2015.

\section{Preliminaries on fractional calculus}

Fractional calculus is an extension of the ordinary calculus, by considering integrals and derivatives of arbitrary real or complex order [33, 59]. This subject is as old as calculus itself, and in the past decades it has proved to be applicable to real world phenomena. In some cases, considering the dynamic being modeled by a fractional derivative/integral, we obtain a more realistic model.

Let $\alpha>0$ be a real number and $x:[a, b] \rightarrow \mathbb{R}$ an integrable function. The Riemann-Liouville fractional integral of $x$ of order $\alpha$ is given by the expression

$$
I_{a+}^{\alpha} x(t):=\frac{1}{\Gamma(\alpha)} \int_{a}^{t}(t-\tau)^{\alpha-1} x(\tau) d \tau
$$

where $\Gamma$ denotes the Gamma function

$$
\Gamma(t):=\int_{0}^{\infty} \tau^{t-1} \exp (-\tau) d \tau
$$

for $t>0$. We remark that $\Gamma(t+1)=t \Gamma(t)$, for all $t>0$, and for positive integers $n$, we have $\Gamma(n+1)=n !$.

Given a function $x:[a, b] \rightarrow \mathbb{R}$ of class $C^{n}$, the Caputo fractional derivative of $x$ of order $\alpha$ is defined by

$$
{ }^{C} D_{a+}^{\alpha} x(t):= \begin{cases}\frac{1}{\Gamma(n-\alpha)} \int_{a}^{t}(t-\tau)^{n-\alpha-1} x^{(n)}(\tau) d \tau, & \text { if } \alpha \notin \mathbb{N}, n=[\alpha]+1 \\ x^{(n)}(t), & \text { if } \alpha=n \in \mathbb{N} .\end{cases}
$$

One important relation between these two fractional operators is stated next [33]:

1. Given a continuous function $x:[a, b] \rightarrow \mathbb{R},{ }^{C} D_{a+}^{\alpha} I_{a+}^{\alpha} x(t)=x(t)$.

2. If $x:[a, b] \rightarrow \mathbb{R}$ is a function of class $C^{n}$, then

$$
I_{a+}^{\alpha}{ }^{C} D_{a+}^{\alpha} x(t)=x(t)-\sum_{k=0}^{n-1} \frac{x^{(k)}(a)}{k !}(t-a)^{k} .
$$

Another function of great importance for the fractional calculus is the Mittag-Leffler function $E_{\alpha}$, which is defined by the series

$$
E_{\alpha}(t):=\sum_{k=0}^{\infty} \frac{t^{k}}{\Gamma(\alpha k+1)}, \quad t \in \mathbb{R}
$$

While the Gamma function is a generalization of the factorial function, the Mittag-Leffler function is a generalization of the exponential function once if $\alpha=1, E_{1}(t)=\exp (t)$. Since, in general, the 
solutions of linear ODE's involve the classical exponential function, the Mittag-Leffler function appears naturally in the solution of fractional order differential equations [33]. We also remark that the Mittag-Leffler function is useful to describe the electrical properties of nerve cell membranes and the propagation of electrical signals [44].

Theorem 1. [13] Let $x$ be a function such that $x$ and ${ }^{C} D_{a+}^{\alpha} x$ are continuous, for $\alpha \in(0,1]$. Then, for all $t \in(a, b]$, there exists some $c \in] a, t$ [ satisfying the condition

$$
x(t)=x(a)+\frac{1}{\Gamma(\alpha+1)}{ }^{C} D_{a+}^{\alpha} x(c)(t-a)^{\alpha} .
$$

Thus, from Theorem 1, we conclude that if ${ }^{C} D_{a+}^{\alpha} x(t)>0$, for all $t \in[a, b]$, then the function $x$ is strictly increasing, and if ${ }^{C} D_{a+}^{\alpha} x(t)<0$, for all $t \in[a, b]$, then the function $x$ is strictly decreasing.

One other useful tool is the Gronwall inequality [67], which plays an important role in stability, boundness, uniqueness, and continuous dependence on the initial or boundary value and parameters of solutions to differential and integral equations. With this tool, we prove the next result that is useful to prove the nonnegativity of solutions for our fractional MSEIR model.

Lemma 1. Let $\alpha \in(0,1), m \in \mathbb{N}$, and denote the vectors $X:=\left(x_{1}, \ldots, x_{m}\right)$ and $Y:=$ $\left(y_{1}, \ldots, y_{m}\right)$. For each $i=1, \ldots, m$, let $f_{i}:[a, b] \times \mathbb{R}^{m} \rightarrow \mathbb{R}$ be a continuous function and Lipschtiz with respect to the second component:

$$
\left|f_{i}(t, X)-f_{i}(t, Y)\right| \leq L_{i}\|X-Y\|,
$$

where $L_{i}$ is a constant. Let us denote $f:=\left(f_{1}, \ldots, f_{m}\right)$, and consider the two fractional differential equations

$$
{ }^{C} D_{a+}^{\alpha} X(t)=f(t, X)+\frac{1}{k} \quad \text { and } \quad{ }^{C} D_{a+}^{\alpha} X(t)=f(t, X),
$$

with the same initial conditions, where $k$ is a positive integer. If ${ }_{k} X^{\star}:=\left({ }_{k} x_{1}^{*}, \ldots,{ }_{k} x_{m}^{*}\right)$ and $X^{\star}:=\left(x_{1}^{*}, \ldots, x_{m}^{*}\right)$ are the solutions of $(1)$, respectively, then ${ }_{k} X^{\star}(t) \rightarrow X^{\star}(t)$ as $k$ goes to infinity, for all $t \in[a, b]$.

Proof. Applying the fractional integral to both sides of Eqs. (1), we get that

$$
\begin{aligned}
\left\|_{k} X^{\star}(t)-X^{\star}(t)\right\| & \leq \sum_{i=1}^{m}\left|{ }_{k} x_{i}^{\star}(t)-x_{i}^{\star}(t)\right| \\
& \leq \sum_{i=1}^{m}\left[\frac{1}{\Gamma(\alpha)} \int_{a}^{t}(t-\tau)^{\alpha-1}\left|f_{i}\left(\tau,{ }_{k} X^{\star}(\tau)\right)-f_{i}\left(\tau, X^{\star}(\tau)\right)\right| d \tau+\frac{(t-a)^{\alpha}}{\Gamma(\alpha+1) k}\right] \\
& \leq \sum_{i=1}^{m}\left[\frac{L_{i}}{\Gamma(\alpha)} \int_{a}^{t}(t-\tau)^{\alpha-1}\left\|_{k} X^{\star}(\tau)-X^{\star}(\tau)\right\| d \tau+\frac{(t-a)^{\alpha}}{\Gamma(\alpha+1) k}\right] .
\end{aligned}
$$

Using the Gronwall inequality [4, Theorem 8], we conclude that

$$
\left\|_{k} X^{\star}(t)-X^{\star}(t)\right\| \leq \frac{m(t-a)^{\alpha}}{k \Gamma(\alpha+1)} E_{\alpha}\left(\sum_{i=1}^{m} L_{i}(t-a)^{\alpha}\right) .
$$

Thus, as $k \rightarrow \infty$, one has $\left\|_{k} X^{\star}(t)-X^{\star}(t)\right\| \rightarrow 0$.

\section{The classical MSEIR model}

In our work we assume that, once recovered from the disease, the individual gets lifelong immunity. Also, the birth and dead rates are the same, constant in time, and denoted by $b$, so that the total 
population $N=M+S+E+I+R$ is constant along time. The class of susceptible $S$ increases at a rate $b S$, since only mothers that are susceptible can have susceptible newborns (without passive immunity). All other classes were exposed to the disease and have immunity, and so the other newborns $b(N-S)$ enter the passively immune class $M$. Individuals can be transferred from one compartment to another at different rates. By $\delta M$, we mean the transfer out of $M, \mu S$ is the transfer out of $S, \epsilon E$ the transfer out of $E$ and $\gamma I$ the recovery rate from the infectious class (Figure 1 ). For example, for the varicella, the values are $1 / \delta=6$ months, $1 / \epsilon=14$ days, and $l / \gamma=7$ days [62]. Let $\beta$ be the transmission rate, that is, the probability of a susceptible individual to be infected after an effective contact with one infectious individual, so that $\mu=\beta I / N$.

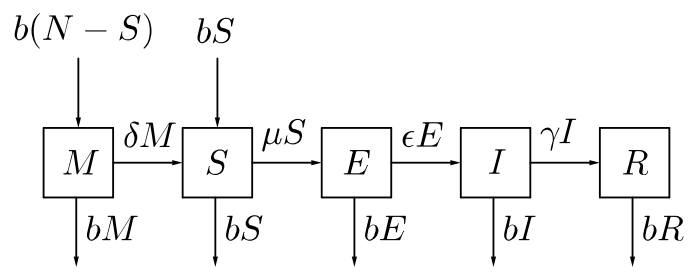

Figure 1: The MSEIR model.

The flow of the infection is given by the following system of ordinary differential equations (cf. [20]):

$$
\left\{\begin{aligned}
M^{\prime}(t) & =b(N-S(t))-(\delta+b) M(t) \\
S^{\prime}(t) & =b S(t)+\delta M(t)-\frac{\beta}{N} S(t) I(t)-b S(t) \\
E^{\prime}(t) & =\frac{\beta}{N} S(t) I(t)-(\epsilon+b) E(t) \\
I^{\prime}(t) & =\epsilon E(t)-(\gamma+b) I(t) \\
R^{\prime}(t) & =\gamma I(t)-b R(t) .
\end{aligned}\right.
$$

The variable $t$ represents time, and can be measured in hours, days, weeks, or months, depending on the disease spread. Doing the substitution $S=N-M-E-I-R$, we can remove the differential equation for $S$, and considering $m:=M / N, e:=E / N, i:=I / N$, and $r:=R / N$, we obtain the simpler model

$$
\begin{cases}m^{\prime}(t) & =b(e(t)+i(t)+r(t))-\delta m(t) \\ e^{\prime}(t) & =\beta i(t)(1-m(t)-e(t)-i(t)-r(t))-(\epsilon+b) e(t) \\ i^{\prime}(t) & =\epsilon e(t)-(\gamma+b) i(t) \\ r^{\prime}(t) & =\gamma i(t)-b r(t)\end{cases}
$$

\section{A fractional MSEIR model}

Motivated by system (2), we present here our fractional MSEIR model. First, we replace each ordinary derivative in the system by the Caputo fractional derivative of order $\alpha$, where $\alpha$ is an arbitrary real belonging to the interval $(0,1)$. Then, each parameter $\star$ is replaced by $\star^{\alpha}$, in order to both sides of the equations have the same dimension, with the exception of $N$, that is dimensionless [14]. Therefore, our proposal model is given by the following system of nonlinear fractional differential equations:

$$
\begin{cases}{ }^{C} D_{0+}^{\alpha} M(t) & =b^{\alpha}(N-S(t))-\left(\delta^{\alpha}+b^{\alpha}\right) M(t) \\ { }^{C} D_{0+}^{\alpha} S(t) & =b^{\alpha} S(t)+\delta^{\alpha} M(t)-\frac{\beta^{\alpha}}{N} S(t) I(t)-b^{\alpha} S(t) \\ { }^{C} D_{0+}^{\alpha} E(t) & =\frac{\beta^{\alpha}}{N} S(t) I(t)-\left(\epsilon^{\alpha}+b^{\alpha}\right) E(t) \\ { }^{C} D_{0+}^{\alpha} I(t) & =\epsilon^{\alpha} E(t)-\left(\gamma^{\alpha}+b^{\alpha}\right) I(t) \\ { }^{C} D_{0+}^{\alpha} R(t) & =\gamma^{\alpha} I(t)-b^{\alpha} R(t) .\end{cases}
$$


It is assumed that $M, S, E, I, R$ and their Caputo fractional derivatives are continuous functions. Similarly to the classical case, we have ${ }^{C} D_{0+}^{\alpha} N(t)={ }^{C} D_{0+}^{\alpha}(M+S+E+I+R)(t)=0$, and so we conclude that $M+S+E+I+R$ is constant, and equal to the population size. We can rewrite the fractional system (4), and obtain the following one:

$$
\begin{cases}{ }^{C} D_{0+}^{\alpha} m(t) & =b^{\alpha}(e(t)+i(t)+r(t))-\delta^{\alpha} m(t) \\ { }^{C} D_{0+}^{\alpha} e(t) & =\beta^{\alpha} i(t)(1-m(t)-e(t)-i(t)-r(t))-\left(\epsilon^{\alpha}+b^{\alpha}\right) e(t) \\ { }^{C} D_{0+}^{\alpha} i(t) & =\epsilon^{\alpha} e(t)-\left(\gamma^{\alpha}+b^{\alpha}\right) i(t) \\ { }^{C} D_{0+}^{\alpha} r(t) & =\gamma^{\alpha} i(t)-b^{\alpha} r(t),\end{cases}
$$

with the initial conditions

$$
m(0)=m_{0}, e(0)=e_{0}, i(0)=i_{0} \text { and } r(0)=r_{0},
$$

where $0 \leq m_{0}, e_{0}, i_{0}, r_{0} \leq 1$. The susceptible population is given by $s(t)=1-m(t)-e(t)-i(t)-r(t)$. Remark 1. In $[16,56]$, one other method to deal with the inconsistency of the dimensions between the left and right hand sides of the equations, due to simply replacing ordinary derivatives by fractional derivatives in model (3), is proposed. The procedure consists of multiplying the lefthand size of each equation by $\tau^{\alpha-1}$, where $\tau$ is a time constant used to balance the units, after replacing the differential operators. In such case, the fractional system becomes

$$
\begin{cases}\tau^{\alpha-1 C} D_{0+}^{\alpha} m(t) & =b(e(t)+i(t)+r(t))-\delta m(t) \\ \tau^{\alpha-1 C} D_{0+}^{\alpha} e(t) & =\beta i(t)(1-m(t)-e(t)-i(t)-r(t))-(\epsilon+b) e(t) \\ \tau^{\alpha-1 C} D_{0+}^{\alpha} i(t) & =\epsilon e(t)-(\gamma+b) i(t) \\ \tau^{\alpha-1 C} D_{0+}^{\alpha} r(t) & =\gamma i(t)-b r(t) .\end{cases}
$$

Theorem 2. There is a unique solution for the initial value problem given by (5)-(6) and the solution belongs to $\left(R_{0}^{+}\right)^{4}:=\left\{\left(x_{1}, x_{2}, x_{3}, x_{4}\right) \in \mathbb{R}^{4}: x_{i} \geq 0\right.$, for all $\left.i\right\}$.

Proof. The global existence and uniqueness of the solution follows from Theorem 3.1 and Remark 3.2 of [40], respectively. To show the nonnegativity of the solution, consider the following auxiliary system of fractional differential equations:

$$
\begin{cases}{ }^{C} D_{0+}^{\alpha} m(t) & =b^{\alpha}(e(t)+i(t)+r(t))-\delta^{\alpha} m(t)+1 / k \\ { }^{C} D_{0+}^{\alpha} e(t) & =\beta^{\alpha} i(t)(1-m(t)-e(t)-i(t)-r(t))-\left(\epsilon^{\alpha}+b^{\alpha}\right) e(t)+1 / k \\ { }^{C} D_{0+}^{\alpha} i(t) & =\epsilon^{\alpha} e(t)-\left(\gamma^{\alpha}+b^{\alpha}\right) i(t)+1 / k \\ { }^{C} D_{0+}^{\alpha} r(t) & =\gamma^{\alpha} i(t)-b^{\alpha} r(t)+1 / k\end{cases}
$$

with $k \in \mathbb{N}$. We will prove that solution of $(6)-(7)\left(m_{k}^{\star}(t), e_{k}^{\star}(t), i_{k}^{\star}(t), r_{k}^{\star}(t)\right)$ belongs to $\left(R_{0}^{+}\right)^{4}$, for all $t \geq 0$. In order to obtain a contradiction, let us assume that there exists some instant in time where the condition fails. Let

$$
t_{0}:=\inf \left\{t>0 \mid\left(m_{k}^{\star}(t), e_{k}^{\star}(t), i_{k}^{\star}(t), r_{k}^{\star}(t)\right) \notin\left(R_{0}^{+}\right)^{4}\right\} .
$$

Thus, $\left(m_{k}^{\star}\left(t_{0}\right), e_{k}^{\star}\left(t_{0}\right), i_{k}^{\star}\left(t_{0}\right), r_{k}^{\star}\left(t_{0}\right)\right) \in\left(R_{0}^{+}\right)^{4}$ and one of the quantities $m_{k}^{\star}\left(t_{0}\right), e_{k}^{\star}\left(t_{0}\right), i_{k}^{\star}\left(t_{0}\right)$ or $r_{k}^{\star}\left(t_{0}\right)$ is zero. Suppose that $m_{k}^{\star}\left(t_{0}\right)=0$. Since

$$
{ }^{C} D_{0+}^{\alpha} m_{k}^{\star}\left(t_{0}\right)=b^{\alpha}\left(e_{k}^{\star}\left(t_{0}\right)+i_{k}^{\star}\left(t_{0}\right)+r_{k}^{\star}\left(t_{0}\right)\right)+\frac{1}{k}>0,
$$

by continuity of ${ }^{C} D_{0+}^{\alpha} m_{k}^{\star}$, we conclude that ${ }^{C} D_{0+}^{\alpha} m_{k}^{\star}\left(\left[t_{0}, t_{0}+\zeta[) \subseteq \mathbb{R}^{+}\right.\right.$, for some $\zeta>0$. By Theorem $1, m_{k}^{\star}\left(\left[t_{0}, t_{0}+\zeta[) \subseteq \mathbb{R}_{0}^{+}\right.\right.$and so $m_{k}^{\star}$ is nonnegative. In an analogous way we can prove that the remaining functions $e_{k}^{\star}, i_{k}^{\star}$ and $r_{k}^{\star}$ are nonnegative, obtaining a contradiction. By Lemma 1 , as $k \rightarrow \infty$, we conclude that the solution $\left(m^{\star}(t), e^{\star}(t), i^{\star}(t), r^{\star}(t)\right)$ of (5)-(6) belongs to $\left(R_{0}^{+}\right)^{4}$, for all $t \geq 0$, proving the desired result.

Theorem 3. Model (5)-(6) has at most two equilibrium points: 
1. a disease free equilibrium $P_{F}=(0,1,0,0,0)$, and

2. an endemic equilibrium point $P_{E}=\left(m^{\star}, s^{\star}, e^{\star}, i^{\star}, r^{\star}\right)$, with

$$
s^{\star}=1-m^{\star}-e^{\star}-i^{\star}-r^{\star}, \quad r^{\star}=\frac{\gamma^{\alpha}}{b^{\alpha}} i^{\star}, \quad e^{\star}=\frac{\gamma^{\alpha}+b^{\alpha}}{\epsilon^{\alpha}} i^{\star},
$$

and

$$
\begin{aligned}
m^{\star} & =\frac{b^{\alpha}\left(\beta^{\alpha} \epsilon^{\alpha}-\left(b^{\alpha}+\gamma^{\alpha}\right)\left(b^{\alpha}+\epsilon^{\alpha}\right)\right)}{\epsilon^{\alpha} \beta^{\alpha}\left(b^{\alpha}+\delta^{\alpha}\right)}, \\
i^{\star} & =\frac{b^{\alpha} \delta^{\alpha}\left(\beta^{\alpha} \epsilon^{\alpha}-\left(b^{\alpha}+\gamma^{\alpha}\right)\left(b^{\alpha}+\epsilon^{\alpha}\right)\right)}{\beta^{\alpha}\left(b^{3 \alpha}+b^{2 \alpha}\left(\delta^{\alpha}+\epsilon^{\alpha}+\gamma^{\alpha}\right)+b^{\alpha}\left(\delta^{\alpha} \epsilon^{\alpha}+\delta^{\alpha} \gamma^{\alpha}+\epsilon^{\alpha} \gamma^{\alpha}\right)+\delta^{\alpha} \epsilon^{\alpha} \gamma^{\alpha}\right)},
\end{aligned}
$$

if $m^{\star}, s^{\star}, e^{\star}, i^{\star}, r^{\star}$ are between 0 and 1 .

Proof. The equilibrium points are computed by putting the right hand sides of the four equations given in (5) equal to zero.

Theorem 4. The disease free equilibrium $P_{F}$ of system (5)-(6) is locally asymptotically stable if

$$
\frac{\beta^{\alpha} \epsilon^{\alpha}}{\left(\epsilon^{\alpha}+b^{\alpha}\right)\left(\gamma^{\alpha}+b^{\alpha}\right)}<1
$$

Proof. The Jacobian matrix of (5), evaluated at the equilibrium point $P_{F}$, is the following

$$
\left[\begin{array}{cccc}
-\delta^{\alpha} & b^{\alpha} & b^{\alpha} & b^{\alpha} \\
0 & -\epsilon^{\alpha}-b^{\alpha} & \beta^{\alpha} & 0 \\
0 & \epsilon^{\alpha} & -\gamma^{\alpha}-b^{\alpha} & 0 \\
0 & 0 & \gamma^{\alpha} & -b^{\alpha}
\end{array}\right]
$$

The eigenvalues of matrix (8) are

$$
\lambda=-\delta^{\alpha} \vee \lambda=-b^{\alpha} \vee \lambda=\frac{-(A+B) \pm \sqrt{(A+B)^{2}-4(A B-C)}}{2},
$$

with

$$
A:=\epsilon^{\alpha}+b^{\alpha}, \quad B:=\gamma^{\alpha}+b^{\alpha}, \quad C:=\beta^{\alpha} \epsilon^{\alpha} .
$$

As it was proven in $[3,46]$, the disease free equilibrium for (5) is locally asymptotically stable if all eigenvalues $\lambda_{i}$ of the Jacobian matrix (8) satisfy the following inequality

$$
\left|\arg \left(\lambda_{i}\right)\right|>\alpha \frac{\pi}{2} .
$$

Since $A+B>0$ and $(A+B)^{2}-4(A B-C)>0$, condition (9) is meet if

$$
\frac{\beta^{\alpha} \epsilon^{\alpha}}{\left(\epsilon^{\alpha}+b^{\alpha}\right)\left(\gamma^{\alpha}+b^{\alpha}\right)}<1
$$

proving the desired result.

The number

$$
R_{0}:=\frac{\beta^{\alpha} \epsilon^{\alpha}}{\left(\epsilon^{\alpha}+b^{\alpha}\right)\left(\gamma^{\alpha}+b^{\alpha}\right)}
$$

is called the basic reproduction number. This number represents the average number of secondary infections produced by an infected individual in a susceptible population. 


\section{Numerical simulations / A case study}

In this section, we obtain numerical results for the solution of the fractional MSEIR model and compare it with the results of the classical MSEIR model, applied to an epidemic of varicella that occurred in China in 2015 [65]. We remark that there are no general methods to solve analytically systems of differential equations in the classical case or in the fractional case. In order to obtain approximate numerical solutions of the fractional order model, we have used the Predictor-Corrector approach [15].

It is well known that varicella is a disease commonly affecting children, teens, and young adults and it is spread by direct contact or airborne droplets. Accordingly to Tang et al. [65], between 2010 and 2015, there were two epidemic waves annually among school populations in Shenzhen, China. Furthermore, the authors refer that these outbreaks pose serious health threats to the juvenile population since the Chinese Immunization Program does not cover vaccination against varicella. Note that this problem could be even worse in the near future because the one-child policy, established in 1979, was replaced with the two child-policy since the 29th of October of 2015.

We will use the data from the paper of Tang et al. [65], consisting of the number of weekly varicella confirmations of people living in Shenzhen from 2013 to 2015. The authors used data from the Infectious Disease Reporting Information Management and, in our present work, we considered the data consisting in 22 weeks, counting from the 19th week until the 40th week of 2015 , with a total population of $N=1500000$.

Table I: Data provided by the Infectious Disease Reporting Information Management System

\begin{tabular}{llllllllllll}
\hline Week & 19 & 20 & 21 & 22 & 23 & 24 & 25 & 26 & 27 & 28 & 29 \\
Cases & 628 & 360 & 533 & 429 & 395 & 324 & 261 & 259 & 249 & 247 & 257 \\
\hline Week & 30 & 31 & 32 & 33 & 34 & 35 & 36 & 37 & 38 & 39 & 40 \\
Cases & 242 & 167 & 153 & 154 & 125 & 90 & 97 & 102 & 128 & 141 & 87
\end{tabular}

In 2015, the number of births per year in China (in millions) was 16.4 and the population was 1371 millions. So, the birth rate is 0.01196 per year. Since our unit of time is one week, our model parameters are

$$
b=0.00023, \quad \delta=\frac{1}{26}, \quad \epsilon=\frac{1}{2} \quad \text { and } \quad \gamma=1
$$

Regarding the initial values for the two differential systems (3) and (5), we assume that at the beginning there are no exposed individuals, and the percentage of vaccinated or recovered from a varicella episode is $65 \%$ (cf. [65]). Also, since the life expectancy in China is 76 years and only mothers exposed to the disease can provide antibodies to the newborns during 6 months, we consider $m(0)=(628 / N+0.65) / 152$. So, the initial data are

$$
m(0)=0.0043, \quad e(0)=0, \quad i(0)=\frac{628}{N} \quad \text { and } \quad r(0)=0.65 .
$$

The goal is to estimate the remaining parameters $\alpha$ and $\beta$, in order to better approximate our theoretical model to real data. For that purpose, we use the routine fminsearch from the Matlab Optimization Toolbox. This routine implements the Nelder-Mead optimization algorithm and the objective function used is based on the least square technique. Given a theoretical model $t \mapsto \Psi\left(t, \lambda_{1}, \ldots, \lambda_{n}\right)$, depending on some unknown parameters $\lambda_{1}, \ldots, \lambda_{n}$ and a sequence of data points $\left(t_{0}, x_{0}\right), \ldots,\left(t_{m}, x_{m}\right)$, the goal is to find the values of the parameters for which the error

$$
E:=\sum_{i=0}^{m}\left(\Psi\left(t_{i}, \lambda_{1}, \ldots, \lambda_{n}\right)-x_{i}\right)^{2}
$$

attains a minimum. The results are the following. For the classical model (3), the value of the parameter $\beta$ is $\beta \approx 2.789687$. For the fractional model (5), we need to estimate the best rate $\beta$ 
and the fractional order $\alpha$. The results are

$$
\beta \approx 4.249134 \text { and } \alpha \approx 0.5408709 \text {. }
$$

We would like to emphasise that these are the best values of $\alpha$ and $\beta$ when we apply the fminsearch routine. The fractional and classical errors were approximately $4.040609 \times 10^{2}$ and $5.077711 \times 10^{2}$, respectively.

In Figure 2, we compare the results obtained for both the classical MSEIR model and the fractional MSEIR model that we studied.

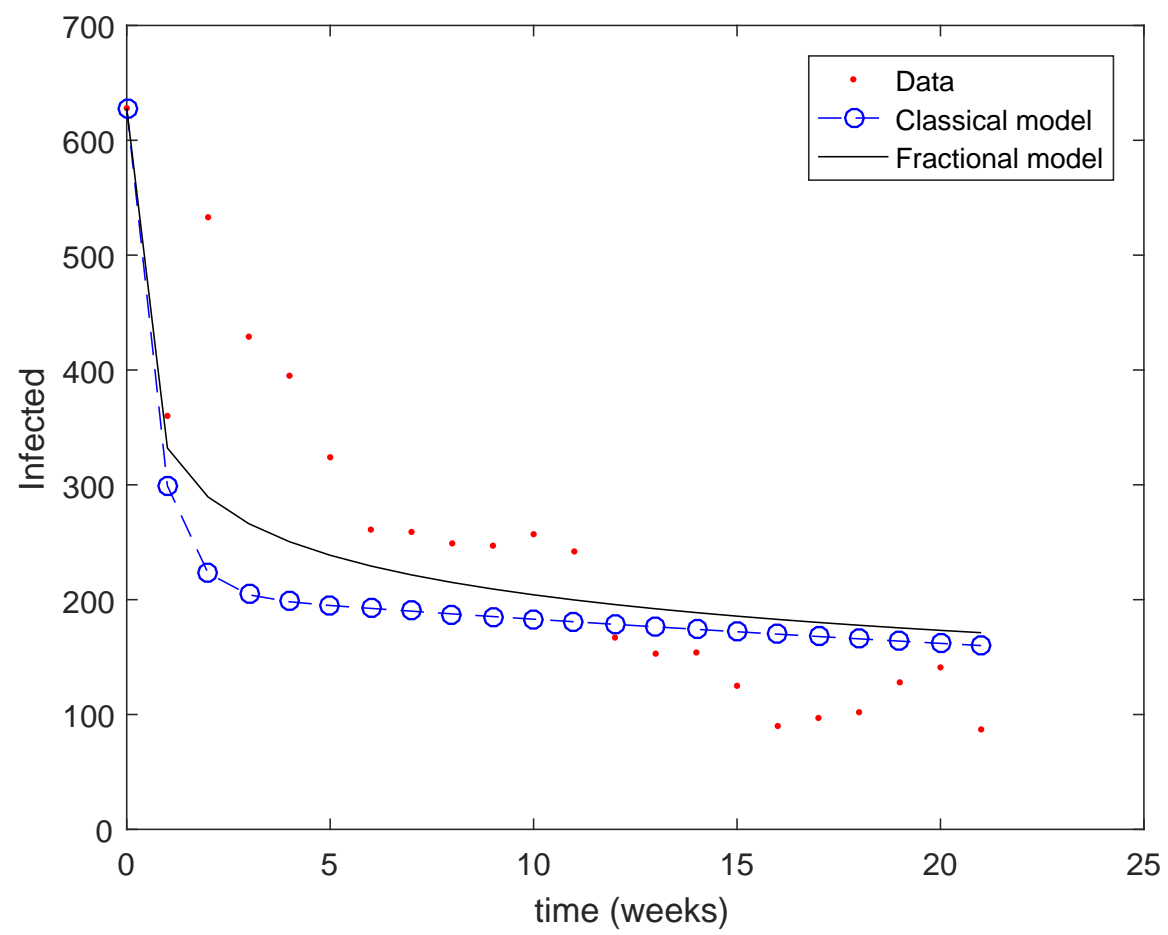

Figure 2: Cases of Varicella in Shenzhen, China, from the 19th until the 40th week of 2015: classical and fractional models.

In this example, the fractional MSEIR model gives us a more accurate fitting to the known real data than the classical MSEIR model. Note that the fractional derivative is a generalization of the classical derivative and for this reason the mathematical models based on fractional differential equations are a more powerful approach than the classical ones. It is also worthwhile to mention that with the fractional approach we need to find the best order $\alpha$ of the fractional derivative that adjusts better to the real data.

\section{Conclusion}

Epidemic models have a major impact on the population worldwide as they provide not only useful information for health authorities in order to understand disease transmission, but also to predict and choose the best strategies to control epidemics.

In this paper, we have proposed a nonlinear fractional order MSEIR model that can be applied to any infectious disease that confers permanent immunity after the individual has been infected by the microorganism causing the disease. We proved that our fractional model has a unique solution and that this solution is nonnegative. We also proved that the fractional order MSEIR model 
has at most two equilibrium points. In addition, a sufficient condition for the local asymptotic stability of disease-free equilibrium was presented.

We tested our fractional MSEIR model with the real data from the population infected with varicella from Shenzhen, China, for the year 2015. We showed that the fractional model best fits the real data when compared to the classic MSEIR model. In fact, the error between the real data and the fractional model decreased approximately $20 \%$.

Finally, it is important to mention that mathematical models based on fractional derivatives are, in general, a more powerful approach to epidemiological models, not only because we can choose the order $\alpha$ of the fractional derivative, but also because of the memory properties of the fractional derivatives.

\section{Acknowledgments}

R. Almeida, A.M.C. Brito da Cruz and N. Martins were supported by Portuguese funds through the CIDMA - Center for Research and Development in Mathematics and Applications, and the Portuguese Foundation for Science and Technology (FCT-Fundação para a Ciência e a Tecnologia), within project UID/MAT/04106/2013; M. T. Monteiro by COMPETE: POCI-01-0145FEDER-007043 and FCT-Fundação para a Ciência e a Tecnologia within the Project Scope: UID/CEC/00319/2013. The authors are grateful to two anonymous referees for valuable comments and suggestions.

(C)2017 The MathWorks, Inc. MATLAB and Simulink are registered trademarks of The MathWorks, Inc. See mathworks.com/trademarks for a list of additional trademarks. Other product or brand names may be trademarks or registered trademarks of their respective holders.

\section{References}

[1] E. Ackerman, L. R. Elveback and J.P. Fox, Simulation of Infectious Disease Epidemics. Charles C. Thomas Publisher, Springfield, IL, 1984.

[2] E. Ahmed, A. Hashish and F. A. Rihan, On fractional order cancer model. Fract. Calc. Appl. Anal. 3 (2012), 1-6.

[3] E. Ahmed, A. M. A. El-Sayed and H. A. A. El-Saka, Equilibrium points, stability and numerical solutions of fractional-order predator-prey and rabies models. J. Math. Anal. Appl. 325 No 1 (2007) 542-553.

[4] R. Almeida, A Gronwall inequality for a general Caputo fractional operator. Math. Inequal. Appl. 20 No 4 (2017) 1089-1105.

[5] R. M. Anderson and R. M. May, Infectious Diseases of Humans: Dynamics and Control. Oxford University Press, 1991.

[6] C. N. Angstmann, B. I. Henry and A. V. McGann, A Fractional Order Recovery SIR Model from a Stochastic Process. Bull Math Biol 78 No 3 (2016) 468-499.

[7] A. J. Arenas, G. González-Parrab and B. M. Chen-Charpentierc, Construction of nonstandard finite difference schemes for the SI and SIR epidemic models of fractional order. Math. Comput. Simul. 121 (2016) 48-63.

[8] H. A. Asfour and M. Ibrahim, On the Differential Fractional Transformation Method of MSEIR Epidemic Model. Int. J. Comput. Appl. 113 No 3 (2015) 10-16.

[9] N. T. J. Bailey, The Mathematical Theory of Infectious Diseases and Its Application. Griffin, London 1975. 
[10] M. Boots and R. Norman, Sublethal infection and the population dynamics of hostmicroparasite interactions. J. Animal Ecology 69 (2000) 517-524.

[11] D. Copot, C. M. Ionescu and R. De Keyser, Relation between fractional order models and diffusion in the body. IFAC Proc. Vol. 47 (3) (2014) 9277-9282.

[12] K. Diethelm. The Analysis of Fractional Differential Equations. Springer-Verlag, Berlin 2010.

[13] K. Diethelm, The mean value theorems and a Nagumo-type uniqueness theorem for Caputo's fractional calculus. Fract. Calc. Appl. Anal. 15 No 2 (2012) 304-313.

[14] K. Diethelm, A fractional calculus based model for the simulation of an outbreak of dengue fever. Nonlinear Dyn 71 No 4 (2013) 613-619.

[15] K. Diethelm, N.J. Ford and A.D. Freed, A Predictor-Corrector Approach for the Numerical Solution of Fractional Differential Equations. Nonlinear Dyn 29 No 3 (2002) 3-22.

[16] A. Dokoumetzidis, R. Magin and P. Macheras, A commentary on fractionalization of multicompartmental models. J Pharmacokinet Pharmacodyn 37 (2010) 203-207.

[17] M. El-Shahed and A. Alsaedi, The Fractional SIRC Model and Influenza A. Math. Probl. Eng. 2011 (2011) Article ID 480378, 9 pages.

[18] M. El-Shahed and F. A. El-Naby, Fractional Calculus Model for Childhood Diseases and Vaccines. Appl. Math. Sci. 8 No 98 (2014) 4859-4866.

[19] L. Esteva and C. Vargas, Analysis of a dengue disease transmission model. Math. Biosciences 150 (1998) 131-151.

[20] H. W. Hethcote, The mathematics of infectious diseases. SIAM Rev. 42 (2000), 599-653.

[21] J. Huo and H. Zhao, Dynamical analysis of a fractional SIR model with birth and death on heterogeneous complex networks. Phys. A 448 (2016) 41-56.

[22] J. F. Gómez-Aguilar, M. G. López-López, V. M. Alvarado-Martínez, D. Baleanu and H. Khan, Chaos in a Cancer Model via Fractional Derivatives with Exponential Decay and Mittag-Leffler Law. Entropy. 19 (2) (2016) 1-19.

[23] G. González-Parra, A. J. Arenas and B. M. Chen-Charpentier, A fractional order epidemic model for the simulation of outbreaks of influenza A(H1N1). Math. Methods Appl. Sci. 37 No 15 (2014) 2218-2226.

[24] E. F. D. Goufo, R. Maritz and J. Munganga, Some properties of Kermack-McKendrick epidemic model with fractional derivative and nonlinear incidence. Adv. Difference Equ. 2014 No 1 (2014), Article ID 278, 9p.

[25] Z. Feng, S. Towers and Y. Yang, Modeling the effects of vaccination and treatment on pandemic influenza. Am. Assoc. Pharm. Sci. J. 13 (3) (2011) 427-437.

[26] J. Glasser D. Taneri, Z. Feng, J.-H. Chuang, P. Tüll, et al, Evaluation of targeted influenza vaccination strategies via population modeling. PLoS One 5 (9) (2010) e12777.

[27] A. Gray, D. Greenhalgh, L. Hu, X. Mao and J. Pan, A stochastic differential equation SIS epidemic model. SIAM J. Appl. Math. 71 (3) (2011), 876-902.

[28] I. Hisashi, Age-structured homogeneous epidemic systems with application to the MSEIR epidemic model. J. Math. Biol. 54 (2007) 101-146.

[29] C. M. Ionescu, A. Lopes, D. Copot, J. A. T. Machado and J. H. T. Bates, The role of fractional calculus in modelling biological phenomena: A review. Commun. Nonlinear Sci. Numer. Simul. 51 (2017) 141-159. 
[30] W. O. Kermack and A. G. McKendrick, A contribution to the mathematical theory of epidemics. Proc. R. Soc. Lond. A 115 (1927) 700-721.

[31] W. O. Kermack and A. G. McKendrick, Contributions to the mathematical theory of epidemics, II - the problem of endemicity. Proc. R. Soc. Lond. A 138 (1932) 55-83.

[32] W. O. Kermack and A. G. McKendrick, Contributions to the mathematical theory of epidemics, III - further studies of the problem of endemicity. Proc. R. Soc. Lond. A 141 (1933) 94-122.

[33] A.A. Kilbas, H.M. Srivastava and J.J. Trujillo, Theory and Applications of Fractional Differential Equations. North-Holland Mathematics Studies, 204. Elsevier Science B.V., Amsterdam, 2006.

[34] A. Korobeinikov, Lyapunov functions and global stability for SIR and SIRS epidemiological models with non-linear transmission, Bull. Math. Biol. 68 No 3 (2006), 615-626.

[35] A. Korobeinikov and P.K. Maini, A Lyapunov function and global properties for SIR and SEIR epidemiological models with nonlinear incidence, Math. Biosci. Eng. 1 No 1 (2004), $57-60$.

[36] R. Kumar and S. Kumar, A New Fractional Modelling on Susceptible-Infected-Recovered Equations with Constant Vaccination Rate. Nonlinear Engrg. 3 No 1 (2013) 11-19.

[37] V. P. Latha, F. A. Rihan, R. Rakkiyappan and G. Velmurugan, A fractional-order delay differential model for Ebola infection and CD8 T-cells response: stability analysis and Hopf bifurcation Int. J. Biomath. 10 (2017), 1750111.

[38] V. P. Latha, F. A. Rihan, R. Rakkiyappan and G. Velmurugan, A fractional-order model for Ebola virus infection with delayed immune response on heterogeneous complex networks. J. Comput. Appl. Math. 339 (2018) 134-146.

[39] M.Y. Li, J.R. Graef, L. Wang and J. Karsai, Global dynamics of a SEIR model with varying total population size. Math. Biosci. 160 (2) (1999), 191-213.

[40] W. Lin, Global existence theory and chaos control of fractional differential equations. J. Math. Anal. Appl. 332 No 1 (2007) 709-726.

[41] M. Lipsitch, T. Cohen, M. Muray and B. R. Levin, Antiviral resistance and the control of pandemic influenza. PLoS Med. 4 (2007) 01110120.

[42] W. Liu, S.A. Levin and Y. Iwasa, Influence of nonlinear incidence rates upon the behavior of SIRS epidemiological models. J. Math. Biol. 23 (2) (1986), 187-204.

[43] G. MacDonald, The Epidemiology and Control of Malaria. Oxford University Press, London, 1957

[44] R. L. Magin, Fractional Calculus in Bioengineering. Begell House, Connecticut 2006.

[45] R. L. Magin, Fractional calculus models of complex dynamics in biological tissues. Comput. Math. Appl. 59 (5) (2010) 1586 - 1593.

[46] D. Matignon, Stability results for fractional differential equations with applications to control processing. In: Computational Engineering in Systems Applications (Lille, France) 2 (1996) 963-968.

[47] A. Mouaouine, A. Boukhouima, K. Hattaf and N. Yousfi, A fractional order SIR epidemic model with nonlinear incidence rate. Adv. Difference Equ. 2018 (1) (2018) 160. 
[48] G. Navarro-Guerrero and Y. Tang, Fractional order model reference adaptive control for anesthesia. Internat. J. Adapt. Control Signal Process. 31 (9) (2017) 1350-1360.

[49] F. Ndairou, I. Area, J. Nieto, C. Silva and D. F. M. Torres, Mathematical modeling of Zika disease in pregnant women and newborns with microcephaly in Brazil. Math. Meth. Appl. Sci. (in press).

[50] E. Okyere, F. T. Oduro, S. K. Amponsah, I. K. Dontwi and N. K. Frempong, Fractional Order SIR Model with Constant Population. British J. Math. Comput. Sci. 14 No 2 (2016) $1-12$.

[51] N. Özalp and E. Demirci, A fractional order SEIR model with vertical transmission. Math. Comput. Modelling 54 No 1 (2011) 1-6.

[52] C. M. A. Pinto and A. R. N. Carvalho, A latency fractional order model for HIV dynamics. J. Comput. Appl. Math. 312 (2017) 240-256.

[53] C. M. A. Pinto and J. A. T. Machado, Fractional model for malaria transmission under control strategies. Comput. Math. Appl. 66 (2013) 908-916.

[54] C. M. A. Pinto and J. A. T. Machado, Fractional model for malaria disease, in: Proceedings of the ASME 2013 International Design Engineering Technical Conferences and Computers and Information in Engineering Conference IDETC/CIE 01/2013, 2013.

[55] S. Pooseh, H. S. Rodrigues and D. F. M., Torres, Fractional derivatives in dengue epidemics. In: T. E. Simos, G. Psihoyios, C. Tsitouras and Z. Anastassi, (eds.) Numerical Analysis and Applied Mathematics, ICNAAM, 739-742. American Institute of Physics, Melville (2011).

[56] J. K. Popovic, M. T. Atanackovic, A. S. Pilipovic, M. R. Rapaic and S. Pilipovic, A new approach to the compartmental analysis in pharmacokinetics: fractional time evolution of diclofenac. J Phamacokinet Pharmacodyn 37 (2010) 119-134.

[57] F. A. Rihan, D. Baleanu, S. Lakshmanan and R. Rakkiyappan, On fractional SIRC Model with Salmonella Bacterial Infection. Abst. Appl. Anal. 2014 (2014) Article ID: 136263.

[58] D. Rostamy and E. Mottaghi, Stability analysis of a fractional-order epidemic model with multiple equilibriums. Adv. Differ. Equ. 2016:170 (2016).

[59] S. G. Samko, A. A. Kilbas and O. I. Marichev, Fractional integrals and derivatives, translated from the 1987 Russian original. Gordon and Breach, Yverdon, 1993.

[60] J. P. C. Santos, L. C. Cardoso, E. M. and N. H. T. Lemes, A Fractional-Order Epidemic Model for Bovine Babesiosis Disease and Tick Populations. Abstr. Appl. Anal. 2015, Article ID 729894 (2015) 10 pages.

[61] T. Sardar, S. Rana, S. Bhattacharya, K. Al-Khaled and J. Chattopadhyay, A generic model for a single strain mosquito-transmitted disease with memory on the host and the vector. Math. Biosci. 263 Supplement C (2015) 18-36.

[62] M. C. Schuette and H. W. Hethcote, Modeling the effects of varicella vaccination programs on the incidence of chickenpox and shingles. Bull. Math. Biol. 61 (1999) 1031-1064.

[63] M. El-Shahed and A. Alsaedi, The fractional SIRC model and influenza A. Math. Probl. Eng. 3 (2011) 378-387.

[64] H. Al-Sulami, M. El-Shahed, J. J. Nieto and W. Shammak, On fractional order Dengue Epidemic Model. Math. Probl. Eng. 2014 (2014) Article ID: 456537. 
[65] X. Tang, S. Zhao, A.P.Y. Chiu, H. Ma, X. Xie, S. Mei, D. Kong, Y. Qin, Z. Chen, X. Wang, D. He, Modelling the transmission and control strategies of varicella among school children in Shenzhen, China. PLoS ONE 12 (5) (2017) 1-17.

[66] R. W. West and J.R. Thompson, Models for the simple epidemic. Math. Biosci. 141 (1997) 29-39.

[67] H. Ye, J. Gao and Y. Ding, A generalized Gronwall inequality and its application to a fractional differential equation. J. Math. Anal. Appl. 328 No 2 (2007) 1075-1081.

[68] Z. U. A. Zafar, K. Rehan and M. Mushtaq, HIV/AIDS epidemic fractional-order model. J. Difference Equ. Appl. 23 (7) (2017) 1298-1315.

[69] X. Zhang and X. Liu, Backward bifurcation and global dynamics of an SIS epidemic model with general incidence rate and treatment. Nonlinear Anal. 2 (2009), 565-575. 